\title{
Klimakrise: Fünf Chancen einer sozial-ökologischen Wende für die Freie Wohlfahrtspflege
}

\section{JONAS PIEPER}

Referent für übergreifende Fachfragen beim Paritätischen Gesamtverband.

https://www.der-paritaetische.de

\author{
Der Klimawandel stellt unsere Gesellschaft, nein: \\ die Menschheit weltweit, vor riesige Herausforderungen. \\ Auch die Freie Wohlfahrtspflege ist davon betroffen.
}

\begin{abstract}
Als Verbraucherin von Ressourcen trägt die Wohlfahrtspflege Verantwortung für ein klimaschonendes Wirtschaften, in der Sozialen Arbeit unterstützt sie die Verwundbarsten, die auch von den Folgen des Klimawandels besonders betroffen sind, und mit ihrer öffentlichen Stimme setzt sie sich für eine soziale Ausgestaltung des Klimaschutzes ein. Denn der notwendige ökologische Wandel kann nur als sozial-ökologischer klappen. Die sozial-ökologische Wende ist eine riesige Herausforderung, sie birgt aber auch neue Chancen für die Gestaltung einer guten, lebenswerten und nachhaltigen Gesellschaft.
\end{abstract}

\section{Drastischer Klimaschutz mit sozialem Ausgleich ist unausweichlich}

Der weltweite Ausstoß von Treibhausgasen ist in den vergangenen Jahrzehnten so massiv gestiegen, dass wir uns mit rasanter Geschwindigkeit auf jene planetare Grenzen zubewegen, in denen die Erde ein noch einigermaßen bewohnbarer Ort ist. Bereits heute sind die Folgen der Erderwärmung spürbar: Das vergangene Jahrzehnt war weltweit das heißeste Jahrzehnt seit Beginn der Wetteraufzeichnungen, seit den 1980ern war jedes Jahrzehnt wärmer als das vorherige. ${ }^{1}$ Weltweit, aber auch hier in Deutschland erleben wir vermehrt Hitzewellen, Dürren, Waldbrände und Starkniederschläge.

Um Schlimmeres abzuwenden und die Erwärmung der Erde auf ein erträg- liches Maß zu begrenzen, muss sich die Art wie wir leben und wirtschaften, grundlegend ändern. Diese Veränderung muss radikal sein - im wahrsten Sinne des Wortes, indem wir unsere fossile Lebensweise von Grund auf umstellen. Wir werden »in sehr kurzer Zeit eine infrastrukturelle, kulturelle, geistige und technische Revolution durchführen müssen, die größte und tiefste seit dem Zweiten Weltkrieg «. ${ }^{2}$ Und tatsächlich gibt es ja auch eine immer stärker werdende und breit getragene gesellschaftliche Bewegung für eine solche gesellschaftliche Transformation, für einen konsequenten Klimaschutz. Spätestens seit dem Sommer 2018 mit seiner außerordentlichen Hitze und dank der Fridays for Future-Bewegung ist die nahende Klimakrise im Zentrum der politischen Debatte angekommen. Dabei wird klar, dass mit wirksamem Klimaschutz auch Kosten verbunden sind und die Frage, wer diese tragen kann und sollte, eine zentral sozialpolitische ist. Wird Klimaschutz, sei es der Abschied aus der fossilen Energiegewinnung, die Bepreisung von $\mathrm{CO}_{2}$ oder die energetische Sanierung von Wohnungen, ohne Blick für bestehende und entstehende Ungleichheiten gemacht, dann belastet er gerade diejenigen, die ohnehin schon wenig haben. Wenn Klimaschutz existentielle Güter wie Nahrung, Wohnen oder Mobilität verteuert ohne einen adäquaten sozialen Ausgleich zu leisten, dann wirkt er spaltend auf diese ohnehin schon sozial ungleiche Gesellschaft. 
All dies macht deutlich: Die notwendige ökologische Wende in unserem gesellschaftlichen Leben und Wirtschaften ist nur als sozial-ökologische denkbar. Klimaschutz braucht eine funktionierende Sozialpolitik. Die sozial-ökologische Wende zu gestalten ist eine riesige Aufgabe. Sie ist die zentrale gesellschaftliche und politische Herausforderung der kommenden Jahre. Dies gilt auch für die Freie Wohlfahrtspflege, für Verbände genauso wie für die praktische Soziale Arbeit. Und es gilt gleich auf zwei Ebenen: Die Verbände sind gefragt, sich gegenüber der Politik für einen Klimaschutz einzusetzen, der den sozialen Ausgleich zu einem zentralen Punkt aller klimapolitischen Vorhaben macht. Als die Bundesregierung im September 2019 mit dem Klimapaket die Einführung eines $\mathrm{CO}_{2}-$ Preises ankündigte, wurde offenbar, wie nötig diese Arbeit der Verbände ist. Die Situation von Menschen in Sozialleistungsbezug oder mit geringen Arbeitslöhnen fand unzureichend bis keine Berücksichtigung. ${ }^{3}$

Neben der politischen Arbeit geht es außerdem darum, die besondere Situation der Sozialen Arbeit vor Ort im Blick zu behalten. Soziale Organisationen sind finanziell häufig so prekär ausgestattet, dass sie überhaupt erst in die Lage versetzt werden müssen, ihr Handeln und Wirtschaften klimafreundlich zu gestalten. Die Einrichtungen und Organisationen der Freien Wohlfahrtspflege sind vielerorts mit großem Einsatz damit beschäftigt, trotz schlechter Rahmenbedingungen eine gute und professionelle Arbeit zu erbringen. Für übergelagerte Themen wie Klimaschutz sind häufig wenig bis keine Ressourcen übrig. Von weitgehenden Maßnahmen, die erhebliche finanzielle Mittel benötigen, ganz zu schweigen: Ohne staatliche Förderung ist es für zahlreiche Kindertagesstätten, Pflegeheime und andere Einrichtungen unmöglich ihre alten Gebäude zu sanieren.

Was nun also tun angesichts der skizzierten Herausforderungen? Sie offensiv, mit Mut und Optimismus angehen. Denn die Freie Wohlfahrtspflege kann nicht nur einiges zu einer klima- und umweltfreundlichen Gesellschaft beitragen - sie kann auch selbst davon profitieren. Mindestens fünf Gründe sprechen dafür.

\section{Die ökologischen Herausforderungen erhöhen die Sensibilität für soziale Ungleichheiten}

Bereits in den Jahren seit der Weltwirtschaftskrise hat die öffentliche Diskussion um soziale Ungleichheit wieder deutlich Fahrt aufgenommen. Die Erkenntnis, dass die Ungleichheit bei Einkommen als auch Vermögen in den vergangenen Jahrzehnten enorm gewachsen ist, dass sich Armut bei einer nicht kleinen Gruppe von Menschen im Lebenslauf verfestigt und dass ein segregierendes Bildungssystem große Hürden für soziale Mobilität geschaffen hat, trägt weiter zur Revitalisierung der Ungleichheitsdebatte bei.

Die ökologischen Herausforderungen verstärken diese Notwendigkeit noch. Denn die entstehenden Kosten für den ökologischen Umbau treffen auf sehr ungleich verteilte Einkommen und Vermögen und auf nachvollziehbare Ängste vor dem wirtschaftlichen Strukturwandel. Diese Erkenntnis wurde politisch zuletzt nicht selten als Grund herangeführt, die klimapolitischen Maßnahmen nicht der Größe der Herausforderungen anzupassen, vor die der Klimawandel uns stellt. Um es klar zu sagen: Hier wurde, mal mehr, mal weniger explizit, Soziales gegen das Umweltthema ausgespielt. Um die ökologischen wie sozialen Herausforderungen zu bewältigen, dürfen sozialpolitische Versäumnisse jedoch keine Ausrede für mangelhaften Klimaschutz sein. Und andersherum gilt: Wirksamer Klimaschutz muss so gestaltet sein, dass diejenigen die Kosten tragen, die dazu auch in der Lage sind. Die Investitionsbedarfe, die auf unsere Gesellschaft zukommen, sind erheblich. Höhere Einnahmen des Staates sind deshalb eine Voraussetzung für das Gelingen der sozial-ökologischen Wende. Damit dies sozial, solidarisch und gerecht stattfindet, müssen sehr hohe Einkommen, große Vermögen und Erbschaften herangezogen werden. Steuerbetrug und Steuervermeidung müssen bekämpft und klamme Kommunen entlastet werden. Für eine soziale Ausgestaltung der notwendigen ökologischen Wende braucht es breite Bündnisse. Und tatsächlich können wir Erstaunliches beobachten: Die soziale Frage taucht zunehmend prominent in ganz neuen Zusammenhängen auf. In der Umweltbewegung beispielsweise werden Fragen nach den sozialen Folgen ökologischer Maßnahmen immer stärker diskutiert und als ein zentraler Faktor für die Lösung ökologischer Probleme anerkannt. So ergeben sich neue Möglichkeiten für gesellschaftliche Bündnisse. Die soziale und die ökologische Frage wird in $\mathrm{Zu}$ kunft nicht mehr getrennt voneinander zu diskutieren sein.

\section{Mobilität wird neu gedacht werden: inklusiv und umweltschonend}

Im Bereich der Mobilität bestehen große Chancen, ökologische und soziale Probleme gemeinsam anzugehen. Aktuell ist der Verkehr in Deutschland weder am Umweltschutz ausgerichtet, noch ist für alle Menschen Mobilität sichergestellt. Die Dominanz des motorisierten Individualverkehrs ist ausgesprochen klimaschädlich. Zugleich stehen Menschen im ländlichen Raum, Geringverdiener*innen oder Sozialleistungsbeziehende vor erheblichen Mobilitätsproblemen. Und schließlich müssen wir in nahezu allen Verkehrsbereichen erhebliche Barrieren für Menschen mit Behinderung feststellen. Teil einer sozial-ökologischen Wende muss es sein, diese Probleme gemeinsam zu lösen. Ein inklusiver, ökologischer und möglichst kostenloser öffentlicher Nahverkehr wäre Kernstück eines solchen Vorhabens. Der Schienenverkehr insgesamt, ob nah oder fern, sollte das gesamte Land in der Fläche erschließen und zu bezahlbaren Preisen möglich sein. Im ländlichen Raum können intelligente Lösungen wie Carsharing ökologische und ökonomische Kosten der Mobilität senken.

Eine derart gestaltete sozial-ökologische Verkehrswende würde für alle Menschen massive Vorteile bringen, insbesondere aber für diejenigen, die besonders darauf angewiesen sind. Sie würde Barrieren für Menschen mit Behinderung abbauen, selbstständig von A nach B zu kommen. Sie würde in den Städten die Luft verbessern und für Kinder und Alte weniger Gefahren auf der Straße bedeuten. Sie wäre mit sinkenden Abgasen ein Beitrag zur Gesundheitsprävention und für bereits Kranke eine Entlastung. Sie würde auf dem Land die Anbindung sicherstellen. Und sie würde Mobilität als eine Grundbedingung für gesellschaftliche Teilhabe sicherstellen. 


\section{Die örtliche und soziale Infrastruktur erlebt eine Revitalisierung}

Kurze Wege zum Bäcker, zur Reinigung oder zum Jugendtreff erhöhen die Lebensqualität und schützen das Klima. Die Gestaltung von Stadtteilen, Quartieren und Dörfern ist damit eine gleichermaßen soziale wie ökologische Aufgabe. Soziale Anlaufpunkte wie Kindergärten, Schulen und Senior"innentreffs, aber auch Ärzt*innen, Einkaufsläden oder Dienstleistungen verringern Fahrtwege und Verkehr. Teil einer sozial-ökologischen Wende muss deshalb die Revitalisierung der regionalen gemeinnützigen und öffentlichen Daseinsvorsorge sein. Sie trägt zur Lebensqualität aller bei und ist klimapolitisch wirkungsvoll. Dazu müssen Mietverhältnisse von sozialen Trägern, kleinen Geschäften und anderen Angeboten wie Gastronomie, Reinigung und andere geschützt werden. Verschuldete Kommunen müssen außerdem in die Lage versetzt werden, sich um die Förderung der örtlichen und sozialen Infrastruktur zu kümmern.

\section{Neue Engagierte mit sozial-ökologischer Motivation tauchen auf}

Mit Fridays for Future ist endgültig sichtbar geworden, wie sehr die aktuelle Generation von Kindern, Jugendlichen und jungen Erwachsenen politisch und gesellschaftlich engagiert ist. Die freitäglichen Demonstrationen an hunderten Orten alleine in Deutschland gipfelten 2019 in mehreren globalen Großstreiks. Initiiert von Fridays for Future und unterstützt durch andere Akteure, gingen beispielsweise am 20. September 2019 in Deutschland mehr als eine Million Menschen auf die Straße, um für einen wirksamen Klimaschutz zu protestieren.

Das Engagement dieser Generation ist thematisch aber keineswegs auf Umweltfragen beschränkt. Studien verweisen auf ein idealistisches junges Milieu, insbesondere in der Altersgruppe von 14 bis 30 Jahren und in der Mehrzahl weiblich, das gleichermaßen eine hohe Sensibilität für ökologische Probleme wie auch für soziale Fragen hat. ${ }^{4}$ Aus diesem Milieu dürfte der Kern der neuen, jungen Umweltbewegung stammen. Unter ihnen herrscht die Überzeugung vor, dass sich unser heutiges Leben und
Wirtschaften grundlegend ändern muss, wenn wir auf diesem Planeten und miteinander dauerhaft ein gutes Leben führen möchten. Junge Menschen aus diesem Milieu engagieren sich für soziale und ökologische Ziele und sie sind bereit, Organisationen aus diesen Bereichen zu unterstützen. ${ }^{5}$

Hier liegt in der sozial-ökologischen Wende eine riesige Chance für soziale Organisationen und Einrichtungen, wenn es der Freien Wohlfahrtspflege gelingt, junge Menschen aus diesem Milieu auch für Engagement im Sozialen zu gewinnen. Thematisch bieten sich hybride Arbeitsfelder an der Schnittstelle von Ökologie und Sozialem, beispielsweise der Beitrag des Nachbarschaftshauses $\mathrm{zu}$ einem nachhaltigen Stadtteil, der integrative Ökohof oder das RepairCafé, genauso an wie die um ökologische Themen erweitere Soziale Arbeit in Einrichtungen. Kitas, Schulen, Jugendtreffs, aber auch Pflegeeinrichtungen, Integrationsangebote oder die Gefährdetenhilfe können ökologische Aspekte, das Verständnis für Natur und Umwelt, in ihre professionelle Arbeit integrieren und sich so für junge Engagierte aus dem sozial-ökologischen Milieu noch stärker öffnen.

\section{Gemeinwohl gewinnt neu an Bedeutung}

Die aktuelle Art des Wirtschaftens, wie sie im weit überwiegenden Teil des Planeten praktiziert wird, ein Wirtschaften, das auf die Ausbeutung von natürlichen und menschlichen Ressourcen setzt, kommt ganz offensichtlich an seine ökologischen, aber auch sozialen Grenzen. Unbestreitbar hat technischer Fortschritt in den vergangenen Jahrzehnten zu einer effizienteren Nutzung von natürlichen Ressourcen geführt. Gleichzeitig ist jedoch die Produktion von Waren so sehr gestiegen, dass insgesamt die Ausbeutung von Ressourcen noch zugenommen hat. Mit dem Earth Overshoot Day wird jährlich angegeben, wann wir als Menschen alle natürlichen Ressourcen aufgebraucht haben, die die Erde in einem Jahr regenerieren kann. Da der Verbrauch über die Jahrzehnte immer weiter zugenommen hat, rückt der Earth Overshoot Day jährlich näher an den Jahresanfang. Lag der Tag Anfang der 1970er Jahre noch im Dezember, war er bis 2019 auf den 29. Juli vorgerückt. ${ }^{6}$
Wenn der Grad der Ausbeutung menschlicher Ressourcen sich auch nicht wie bei natürlichen Ressourcen naturwissenschaftlich genau festlegen lässt, so sind die Zusammenhänge von Wohlstand und Armut zwischen dem globalen Süden und globalen Norden, aber ebenso innerhalb einzelner Gesellschaften, doch sehr präzise beschrieben. Mögen die härtesten gesundheitsschädlichen und zerstörerischen Arbeits- und Lebensbedingungen hierzulande durch sozialen Fortschritt wie die Einführung des Sozialstaates in den vergangenen eineinhalb Jahrhunderten zurückgedrängt worden sein, so lagern wir als hochindustrialisierte "Externalisierungsgesellschaft ${ }^{7}$ die negativen Effekte inzwischen auf ärmere, weniger entwickelte Regionen aus. Der vergleichsweise große - wenn auch ungleich verteilte - Reichtum hier, hängt mit der Armut dort ursächlich zusammen. Doch auch hierzulande können wir angesichts der steigenden Zahl von Geringverdiener*innen und 12,8 Millionen Menschen, die unterhalb der Armutsgrenze leben müssen, kaum von einer sozial nachhaltigen Arbeits- und Lebensweise sprechen. ${ }^{8}$

Elementarer Teil einer sozial-ökologischen Wende muss es angesichts dieser Entwicklungen sein, das aktuell vorherrschende Wirtschaftsparadigma in Frage zu stellen. Das Gemeinwohl mit seinen ökologischen und sozialen Aspekten muss ins Zentrum des Wirtschaftens rücken. In diesem Prozess, in dieser aktuell schon begonnenen, aber umkämpften Transformation, kann die Freie Wohlfahrtspflege richtungsweisend sein. Wenn sie sich auf ihre Stärken der Gemeinnützigkeit besinnt und auch die Versuche einer Vermarktlichung des Sozialen kritisch reflektiert und ihnen eine klare Absage erteilt, kann sie im Prozess der sozial-ökologischen Wende wertvolle Impulse für eine neue Orientierung am Gemeinwohl geben.

\section{Anmerkungen}

(1) World Meteorological Organization (2020): WMO confirms 2019 as second hottest year on record. Pressemitteilung vom 15. Januar 2020. Genf.

(2) Bernd Ulrich (2019): Alles wird anders. Das Zeitalter der Ökologie, Köln: KiWi-Paperback, S. 49. 
(3) Mit der »Sozialplattform Klimaschutz«, initiiert vom Paritätischen Gesamtverband, ver.di, AWO, Caritas, SoVD, VdK, Volkssolidarität und Deutschem Mieterbund, fand sich im Herbst 2019 ein Bündnis zusammen, das sich gegenüber der Politik und in der Öffentlichkeit für eine sozial-ökologische Wende starkmacht. Die Forderungen des Bündnisses reichen von der Wohnungspolitik über Energiekosten für Geringverdienende und Menschen in Sozialleistungsbezug bis zu neuen
Mobilitätskonzepten. Sie greifen das Thema örtliche Infrastruktur genauso auf wie eine sozialpolitische Gesamtstrategie, die in Umbruchszeiten Sicherheit schafft, und schlagen zur Finanzierung der Investitionsbedarfe unter anderem die Heranziehung sehr hoher Einkommen, Vermögen und Erbschaften vor. Mehr unter: http://www.der-paritaetische.de/ schwerpunkt/klimaschutz/

(4) BMU/UBA (2019): Umweltbewusstsein in Deutschland 2018. Berlin/Dessau, S. 80.
(5) Ebd.

(6) Mehr Informationen: www. overshootday.org

(7) Stephan Lessenich (2019): Neben uns die Sintflut. Die Externalisierungsgesellschaft und ihr Preis. München: Piper Taschenbuch.

(8) Der Paritätische (2019): 30 Jahre Mauerfall - Ein viergeteiltes Deutschland. Der Paritätische Armutsbericht 2019. Berlin. Online verfügbar: https://www.der-paritaetische.de/schwerpunkt/armutsbericht/

\section{Wie Digitalisierung zu einer nachhaltigen Entwicklung im Sinne der Agenda 2030 beiträgt}

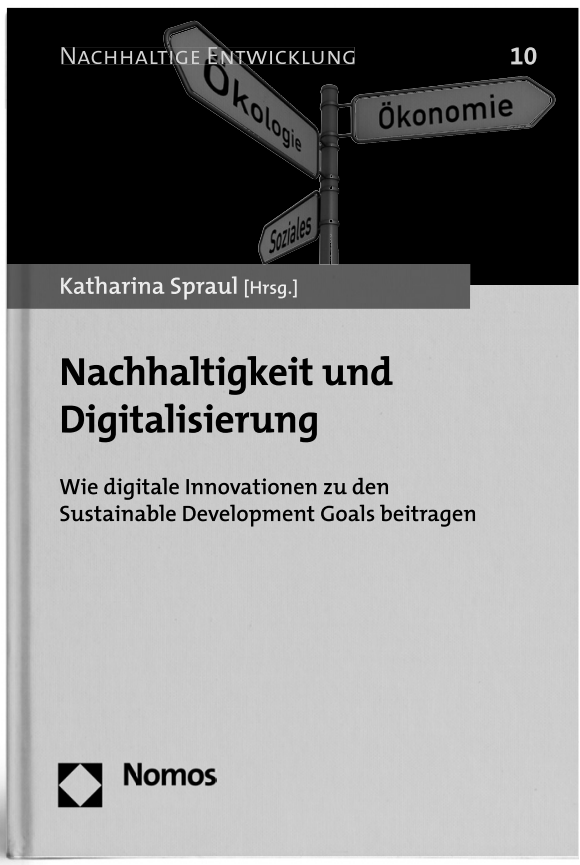

\author{
Nachhaltigkeit und Digitalisierung \\ Wie digitale Innovationen zu den \\ Sustainable Development Goals beitragen \\ Herausgegeben von Prof. Dr. Katharina Spraul \\ 2019, 231 S., brosch., 49, $-€$ \\ ISBN 978-3-8487-6238-5 \\ (Nachhaltige Entwicklung, Bd. 10)
}

Ob Smartphone, Smart Home oder Smart City-die Digitalisierung bestimmt heutzutage fast alle Bereiche unseres Lebens. Auch das Thema der Nachhaltigkeit ist im Jahr 2019 omnipräsent, und es werden zahlreiche damit verbundene Herausforderungen diskutiert. Digitalisierung und Nachhaltigkeit gelten somit als die zwei Megatrends des 21. Jahrhunderts. Inwiefern ist Digitalisierung ein Instrument zur Erreichung einer nachhaltigen Entwicklung? Zur Beantwortung dieser Frage empfehlen sich die von den Vereinten Nationen im Rahmen der Agenda 2030 formulierten Sustainable Development Goals (SDGs). Der Sammelband gibt mit Hilfe empirischer Arbeiten von Studierenden der Technischen Universität Kaiserslautern Antworten auf die Frage, inwiefern verschiedene digitale Technologien zur Erreichung dieser 17 Ziele beitragen.

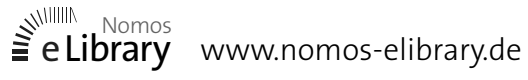

Bestellen Sie im Buchhandel oder versandkostenfrei online unter nomos-shop.de Bestell-Hotline (+49)7221.2104-37 | E-Mail bestellung@nomos.de | Fax (+49)7221.2104-43

Alle Preise inkl. Mehrwertsteuer

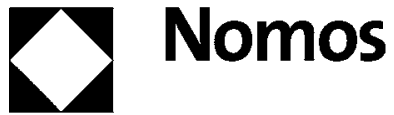

